Bull. Mater. Sci., Vol. 22, No. 3, May 1999, pp. 421-430. (C) Indian Academy of Sciences.

\title{
Linear and nonlinear optical properties of organic crystals: MBANP and its derivatives
}

\author{
R T BAILEY, F R CRUICKSHANK, S LOCHRAN, D PUGH*, J N SHERWOOD, \\ P J LANGLEY ${ }^{\dagger}$ and J D WALLIS ${ }^{\dagger}$ \\ Department of Pure and Applied Chemistry, University of Strathclyde, Glasgow G1 1XL, UK \\ ${ }^{\dagger}$ Centre for Materials Research, School of Physical Sciences, The University of Kent, Canterbury, Kent CT2 7NH, \\ UK
}

\begin{abstract}
In addition to possessing strong optical nonlinearity, the (-)-2-( $\alpha$-methylbenzylamino)-5-nitropyridine (MBANP) crystal has been shown to have unusual linear optical properties. Two methyl derivatives of MBANP have now been synthesized and grown as large single crystals; their refractive indices have been measured as a function of frequency and second-harmonic generation, including the determination of the phase matching loci has been investigated. Data is also available on the racemic form of crystalline MBANP. The properties of this family of crystals have been reviewed and an attempt is made to relate the experimental responses of the crystals to their molecular structure and the calculated properties of the molecules.
\end{abstract}

Keywords. Linear properties; nonlinear; organic crystals; MBANP.

\section{Introduction}

The MBANP, (-)-2-( $\alpha$-methylbenzylamino)-5-nitropyridine, crystal was among a number identified as having strong second order optical nonlinearities (Tweig et al $1982 \mathrm{a}, \mathrm{b}, 1986$ ) in the early 1980's, when there was a major expansion of interest in organic materials for optoelectronics. Subsequently it was shown that, in contrast to many of the materials investigated, it was possible to grow large high quality crystals of MBANP (Bailey et al 1988a, b, 1991a), which could be cut and polished with a view to a complete investigation of the optical properties (Bailey et al 1988c, 1991b). It was soon realized that MBANP exhibited the phenomenon of angular dispersion of the dielectric axes, which had not been observed in any of the other nonlinear optical organic materials (Bailey et al 1988c). Indeed, there is still no other clear-cut example of this phenomenon in an organic crystal that has strong nonlinear optical properties. The occurrence of dispersion of the axes, while it considerably complicates the analysis of the linear and nonlinear optical properties, raises interesting questions concerning the validity of generally accepted rules for determining optimum crystal configurations for phase matching in second-harmonic generation and in other applications (Bailey et al 1993). The disposition of the dielectric axes and refractive indices in MBANP is not particularly favourable to the production of large phasematched second-harmonic generation (SHG) signals. It

*Author for correspondence is possible that other similar compounds may crystallize in more advantageous ways. In view of this possibility and also to investigate further the origins of the rotation of the axes in MBANP it was decided to synthesize a number of derivatives, to identify those that produced non-centrosymmetric crystal structures and to investigate their optical properties. It became apparent that the two derivatives extensively investigated up to the present could also be grown into large, high-quality crystals and it may be that ease of crystal growth is a general feature of this family of molecules, possibly associated with their L-shaped structure and also with a propensity to form strong hydrogen-bonded chains in certain directions (Lochran et al 1998a, b).

Detailed optical measurements on MBANP were also carried out in the late 1980s by Kondo and his coworkers (1988a, b, 1989) who provided, amongst other things, an accurate independent measurement of the principal SHG $d$-coefficient, $d_{22}$, using a smaller cleaved crystal. They also demonstrated how MBANP could be used in a certain type of waveguide. This group has more recently grown noncentrosymmetric racemic crystals of MBANP for which they have complete refractive index and extensive frequency doubling data (Kondo et al 1995). A powder measurement made by Cheng et al (1982) had earlier detected SHG from the racemic material.

At present data are available on structural, linear optical and frequency doubling of four crystals, MBANP, $n$-methyl MBANP, 3-methyl MBANP and the racemate, $( \pm)$ MBANP. The molecular structures, systematic names and crystallographic data are given in figure 1. 


\section{Experimental}

\subsection{The molecular structure}

The structures of the molecules in the MBANP, and $n$-methyl MBANP crystals, as determined by X-ray crystallography, can be seen in figure 2 , where the unit cell is projected onto a plane containing the monoclinic 2 -fold polar axis. The molecule has some conformational freedom associated with rotations about the two single bonds in the bridging group between the pyridine and benzene rings. In MBANP, 3-methyl MBANP, and the racemate the molecular structure is essentially the same with the planes of the two rings nearly at right angles to each other. (This is true of both enantiomers in the racemate). In $N$-methyl MBANP, the presence of the $N$-methyl group prevents the molecule taking up this conformation and a more extended arrangement of the rings is adopted (figure $2 \mathrm{~b}$ ). The asymmetric unit in the $N$-methyl crystal consists of two molecules which have
Compound

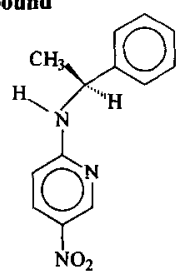

Crystal Strueture

(1) MBANP Monoclinic
$\mathrm{P2},(\mathrm{Z}=2)$

(2) ( $)$ MBANP

Racemic

Orthorhombic

Aba2 $(Z=8)$
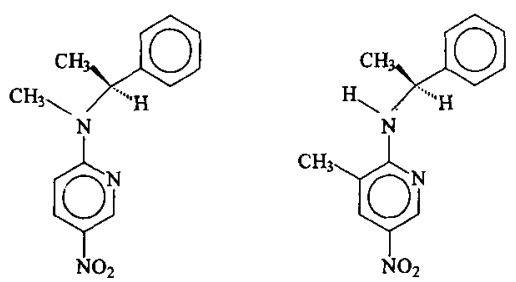

Nomenclature

MBANP:

(-12-( $\alpha$-methylbenzylamino)-5-nitropyridine (common name in NLO literature)

MBANP:

S-5-nitro- N(1-phenylethyl)-2-pyridinamine

n-Methyl MBANP:

S-N-methyl-5-nitro- N-(1-phenylethyl)-2-pyridinamine

3-Methyl MBANP.

S-3-methyl-5-nitro-N-(1-phenylethyl)-2-pyridinamine
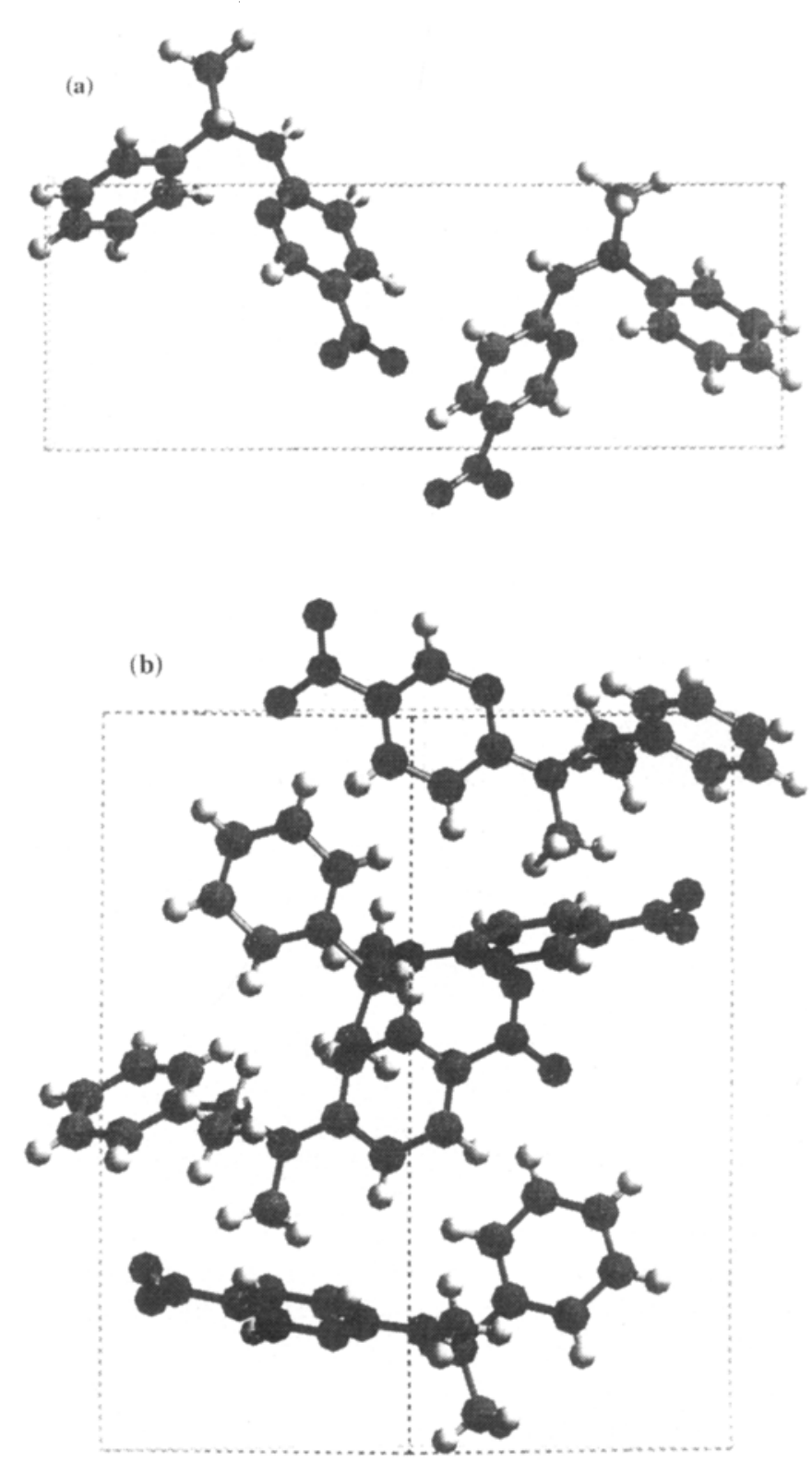

only very slightly differing conformations - both in the more extended form.

Theoretical investigation of the molecular structures using molecular modelling with STO3G and 3-21G $a b$ initio and AM1-MOPAC semi-empirical methods have shown that the above structures and the differences between them are also predicted to occur in the isolated molecules. It is not necessary to invoke the effects of

Figure 2. (a) Projection of the MBANP unit cell onto the bc (100) crystal plane. The monoclinic 2 -fold $\mathbf{b}$ axis points up the page, and (b) projection of the $N$-methyl MBANP unit cell onto the plane containing the monoclinic 2-fold (b) axis and the $z$-dielectric axis. The b-axis points up the page. This plane is intermediate between the two 'molecular planes' (see text), so that the two sets of molecular charge transfer axes are nearly parallel to it. The diagram shows the large angles made between the charge transfer axes and the b-axis.

Figure 1. MBANP and derivatives. 
inter-molecular crystal forces to explain the conformations of the molecules in the crystals.

Electronic structure calculations on the molecule show that the ground state dipole moment is approximately in the direction of the axis joining the nitro and amino nitrogen atoms and that the lowest electronic transitions would have transition moments polarized in this direction. In the usual simplified approach to the theory of the nonlinear response of donor/acceptor organic molecules the charge transfer (CT) axis plays a key role. In the following the molecular $\mathrm{CT}$ axis is always taken to be the axis from the $-\mathrm{NO}_{2}$ nitrogen to the $-\mathrm{NHX}$ amino nitrogen.

\subsection{The crystal structures}

MBANP crystallizes in the polar space group $P 2_{1}$ with two molecules in the unit cell. The monoclinic two-fold axis (b) is the polar axis, with which each of the molecular CT axes make an angle of $37^{\circ}$. This arrangement is shown in figure $2 a$, where the unit cell is projected onto the bc or (100) plane. In figure 3 the projection onto the ac (010) plane is given in conjunction with a stereogram defining some of the important optical properties. There are strongly hydrogen-bonded 'head to tail' chains of molecules extending through the crystal from the positive $(010)$ face to the opposite negative (010) face. These chains do not cross the (001) plane,

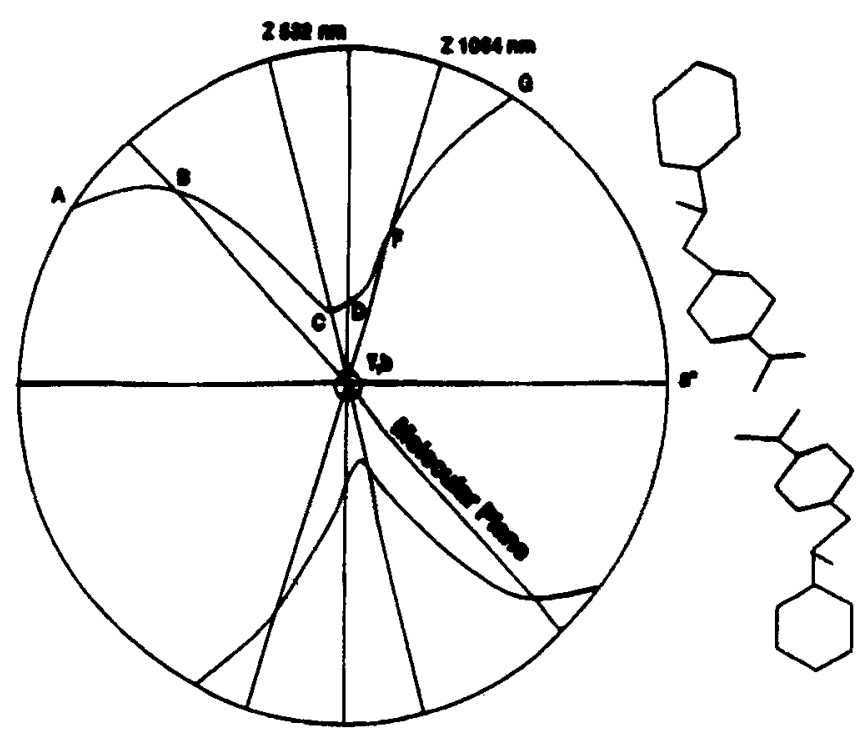

Figure 3. MBANP: Projection onto the ac (010) plane. A, B, $\mathrm{C}, \mathrm{D}, \mathrm{F}$ and $\mathrm{G}$ mark points along the phase matching locus and can be correlated with the plots in figure 6 . The positions of the $z$-dielectric axes at the fundamental and second-harmonic frequencies are also shown. The projection of the two molecules in the unit cell is shown at the side of the stereogram. The line marking the intersection of the molecular plane with the (010) plane is labelled and is parallel to the projections of the molecular CT axes. which has been found experimentally to be the cleavage plane of the crystal.

3-methyl MBANP crystallizes in the noncentrosymmetric, but nonpolar, orthorhombic space group $P 2,2,2$, with four molecules in the unit cell. Hydrogen bonded, 'head to tail' chains similar to those found in MBANP are present, but there are now four chains passing through each unit cell, two with reversed polarity.

$N$-methyl MBANP, like MBANP, crystallizes in the $P 2$, structure, but the asymmetric unit consists of two molecules inclined at different angles to the crystal polar monoclinic 2-fold axis (b). The hydrogen-bonded structure is not so apparent as in the other crystals. The angles between the $\mathrm{NO}_{2}-\mathrm{NHX}$ vector and the polar axis are $80^{\circ}$ and $65^{\circ}$, respectively, in the two components of the asymmetric unit (figure $2 b$ ). The projection of the unit cell onto the ac plane is shown in figure 4 .

The racemic crystal, $( \pm)$ MBANP has been shown (Kondo et al 1995) to have the orthorhombic Aba2 structure with eight molecules in the unit cell. As can

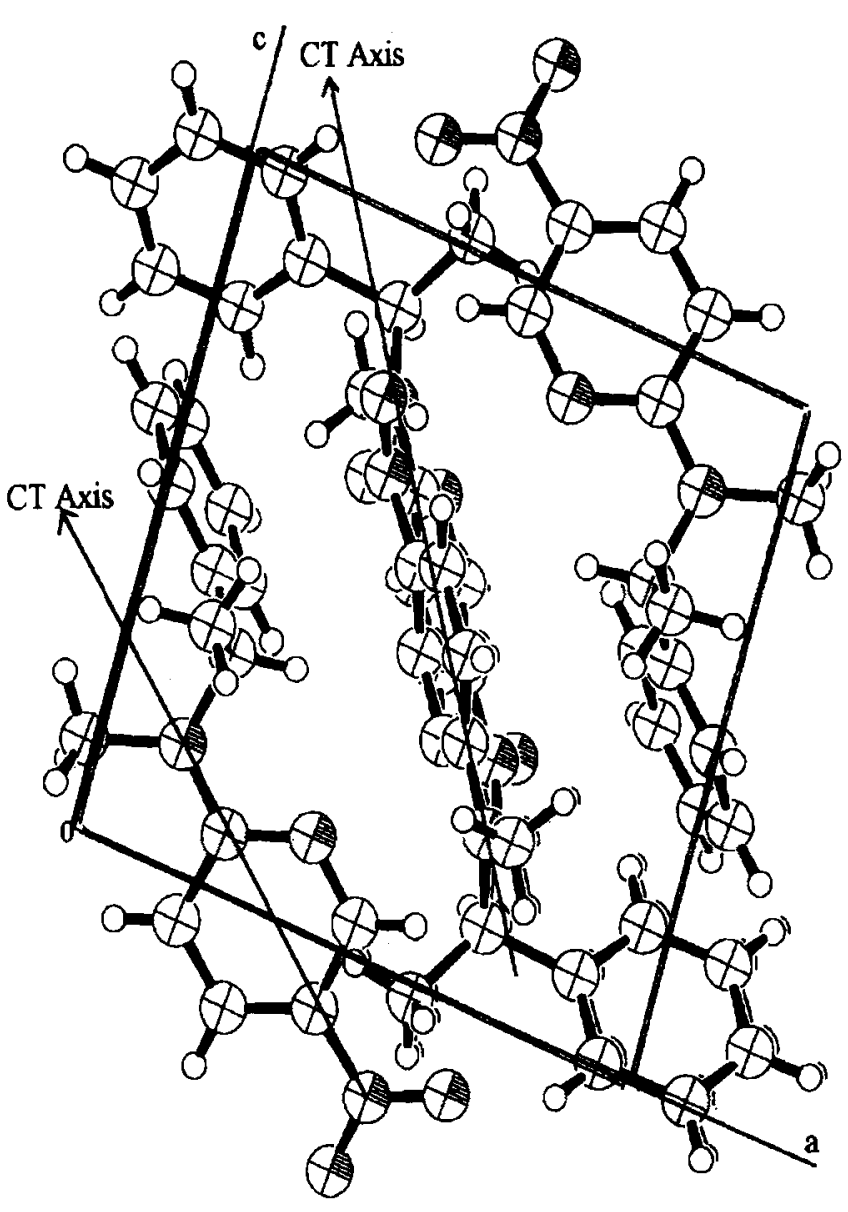

Figure 4. $N$-methyl MBANP: Projection onto the ac (010) plane. The projections of the CT axes of the two molecular components of the asymmetric unit are marked. The $z$-dielectric axis, corresponding to the largest refractive index, approximately bisects the angle between the two CT axes. 
be seen from figure $5 b$ the $c$ axis is a polar axis, but the donor-acceptor vector makes a very large angle with the polar axis and when these vectors are projected onto the ab plane the contributions from the pairs of enantiomers are opposed (figure 5a).

Details of crystal growth and structural characterization, including studies of the defects and perfection of the
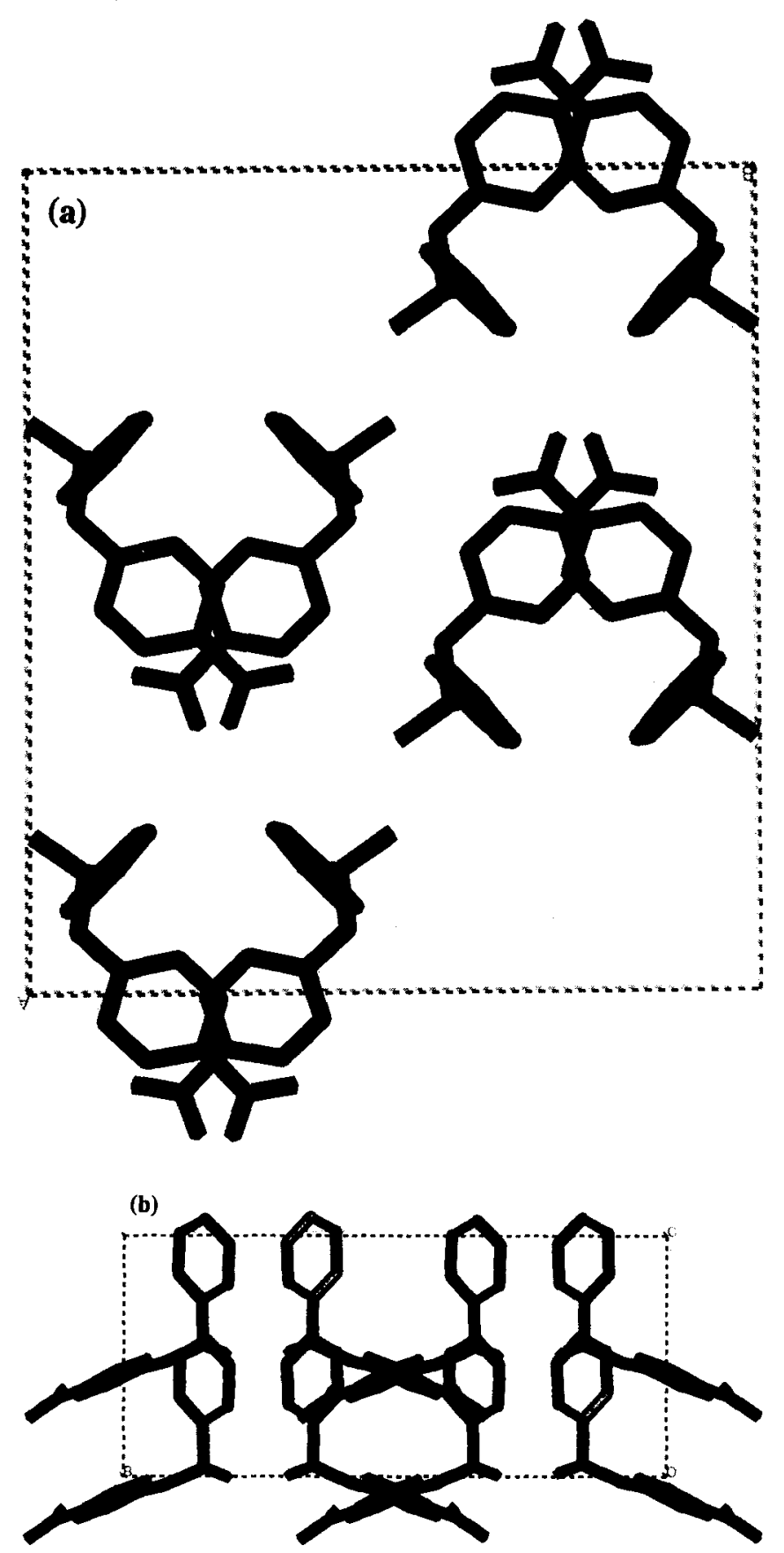

Figure 5. (a) Projection of the racemate, \pm MBANP, unit cell onto the ab (001) plane. The pairs of enantiomers can be identified and the high degree of cancellation of the CT axes is apparent and (b) projection of the racemate, \pm MBANP, unit cell onto the bc (100) plane. The $\mathbf{c}$ axis is a polar axes and points up the page. It can be seen that the noncancelling projections of the CT axes in the c-direction are small. crystals, have been given by the Strathclyde group for MBANP (Bailey et al 1991a) and the two methylated derivatives (Lochran et al 1998a, b) and by the Japanese group for MBANP and the racemate (Kondo et al 1995).

\subsection{Linear optical measurements}

Determinations of the refractive indices as a function of wavelength through the visible have been made on MBANP and the two methylated derivatives by means of deviation measurements on carefully prepared prisms. The technique is described in detail elsewhere (Bailey et al 1993). Values for the highest and lowest principal values for the racemate have been obtained (Kondo et al 1995) using the liquid immersion technique. In all cases Maker fringe spacings have been used to deduce values at the $1.064 \mu \mathrm{m}$ YAG wavelength and in the case of the racemate to provide values of the intermediate refractive indices. The results can be summarized by Sellmeir equations of the general form:

$$
n_{i}^{2}=A+\frac{B \lambda^{2}}{\lambda^{2}-C}-D \lambda^{2}
$$

where $n_{i}$ is one of the principal refractive indices and $\lambda$ the wavelength.

In the case of MBANP a supplementary equation specifying the orientation of the axes in the ac plane as a function of wavelength must also be provided. The Sellmeir equation data are summarized in table 1 , the directions of the dielectric axes for the monoclinic crystals defined in table 2 and explicit values at the fundamental and second-harmonic wavelengths (1064 and $532 \mathrm{~nm})$ for a YAG doubling experiment are given in table 3 .

The relation between the directions of the dielectric axes in the ac plane of the monoclinic crystals and the projections of the molecular structures in the unit cell onto that plane can be examined in figures 3 and 4 .

\subsection{Frequency doubling experiments}

Maker fringes have been measured for a number of different orientations in all the crystals and are the principal source of data for the determination of the components of the d-matrix, which is the conventional two-dimensional representation of the second-order susceptibility tensor for doubling, $\chi_{i j k}(-2 \omega ; \omega, \omega)$. The d-matrix elements as determined by the Maker fringe method are collected in tables 4-7, where they are compared with calculated values. Phase matched SHG has been observed in MBANP and the two methyl derivatives. The phase matching loci can be predicted from the refractive index data and are found to correspond accurately to the observed loci in doubling experiments. The results for Maker fringes and phase matching (MBANP only) are briefly described for each crystal separately. 
2.4a MBANP: Although numerous sets of Maker fringes for different optical orientations have been obtained, it has not been possible to isolate the separate elements of the d-matrix with the exception of the large $d_{22}\left(\chi_{y y y}\right)$ component where all the fields are polarized along the polar monoclinic axis. In all other orientations the rotation of the dielectric axes has the effect of mixing a number of coefficients in the effective coefficient controlling the Maker fringe intensity. In a number of orientations it is impossible to avoid the residual presence of the phase matching signal, which, even in geometries where it is reduced to a few percent of its full value, masks the signal from other nonphase-matched sources.

The value of $d_{22}$ determined by the Maker fringe method (Bailey et al 1988c) is $45 \mathrm{pmV}^{-1}$ (since all fields contributing to $d_{22}$ are aligned with the monoclinic axis the choice of the other two axes does not affect its value). The effective $d$-coefficient for phase matching has a maximum value of $5 \mathrm{pmV}^{-1}$. A stereographic projection of the phase matching locus onto the $(010)$ plane is shown in figure 3 . The intensity varies sharply along the locus and falls to zero at the points $A$ and $G$, as predicted by the form of the $d$-matrix. The maximum value occurs between the points $B$ and $C$ and a local minimum occurs in the neighbourhood of $D$, where the propagation direction is in the bc plane. In a later section it is shown that a model calculation of the response approximates to this behaviour.

Table 1. Sellmeir coefficients.

\begin{tabular}{|c|c|c|c|c|}
\hline & A & B & $\mathrm{C}$ & $\mathrm{D}$ \\
\hline \multicolumn{5}{|c|}{ MBANP } \\
\hline$n_{x}$ & 1.84369 & 0.79567 & 0.06624 & $0 \cdot 02440$ \\
\hline$n_{y}$ & $2 \cdot 37539$ & 0.55690 & $0 \cdot 14067$ & 0.02855 \\
\hline$n_{z}$ & 2.60872 & $0 \cdot 22255$ & 0.16002 & 0.0 \\
\hline \multicolumn{5}{|c|}{ 3-methyl MBANP } \\
\hline$n_{a}$ & 2.78036 & 0.063708 & 0.184186 & $0 \cdot 374153$ \\
\hline$n_{b}$ & $2 \cdot 35431$ & 0.581332 & $0 \cdot 152215$ & 0.009227 \\
\hline$n_{c}$ & 2.63522 & $0 \cdot 153796$ & 0.163522 & 0.081165 \\
\hline \multicolumn{5}{|c|}{$n$-methyl MBANP } \\
\hline$n_{x}$ & $2 \cdot 25903$ & $0 \cdot 117786$ & 0.112279 & 0.026697 \\
\hline$n_{y}$ & 2.51837 & 0.086700 & $0 \cdot 166047$ & 0.148562 \\
\hline$n_{z}$ & 2.68015 & 0.851649 & 0.165316 & -0.043008 \\
\hline \multicolumn{5}{|c|}{ \pm MBANP } \\
\hline$n_{a}$ & $2 \cdot 260$ & 0.550 & 0.1149 & 0 \\
\hline$n_{c}$ & 1.998 & 0.488 & 0.0702 & 0 \\
\hline
\end{tabular}

The table gives the Sellmeir coefficients for use in equation (1). For the orthorhombic crystals $a, b$ and $c$ refer to the crystal axes. For the two monoclinic crystals the dielectric $x, y, z$ axes are defined in table 2 . 2.4b 3-methyl MBANP: The nonpolar orthorhombic structure corresponds to the more symmetric point group 222 , so that the only nonzero elements of the d-matrix are those where all three coordinate indices are different, $d_{14}, d_{25}$ and $d_{36}$. These can be uniquely determined by Maker fringe measurements on three crystal cuts. If Kleinman symmetry were valid, all three should be equal, which is found to be approximately the case. The value found, $\sim 20 \mathrm{pmV}^{-1}$ is reduced by about $50 \%$ compared with the large $d_{22}$ coefficient of MBANP.

2.4c N-methyl MBANP: In this case by making suitable crystal cuts properly oriented with respect to the dielectric axes, which do not change with frequency, it has been possible to make Maker fringe determinations of all the d-matrix elements. Some, however, have much larger experimental errors than others. The space and point groups are the same as in MBANP, but the larger average inclination of the polar axes of the molecules to the polar axis of the crystal removes the dominance of the $d_{22}$ element in the matrix. As a result a number of elements involving coordinate indices in the ac plane might be expected to be comparable. This case therefore provides a rather more stringent test of the theory.

2.4d \pm MBANP: The racemic structure belongs to the point group $m m 2$, again much more symmetric than the monoclinic group. The d-matrix contains six elements which are not zero by crystal symmetry and three of these have been determined by Kondo et al (1995). As would be expected from the orientations of the polar axes of the molecules in the unit cell, where four are opposed to the other four, the values are considerably smaller than those found in the other crystals.

\section{Theoretical interpretation}

\subsection{Linear optical properties}

The contrasting behaviour of the frequency-dependent refractive indices in the two monoclinic crystals requires some explanation.

Table 2. Orientation of the dielectric axes.

MBANP: The z-dielectric axis is obtained by rotating the crystallographic $c$-axis about the positive monoclinic $b$-axis through an angle, $\phi$, given by:

$$
\phi=175.3043-0.43225 \lambda+0.00024 \lambda^{2},
$$

where $\lambda$ is the wavelength in nanometers.

\section{$N$-methyl MBANP:}

The z-dielectric axis is obtained at all optical frequencies by rotating the crystallographic $c$-axis about the positive monoclinic $b$-axis through an angle of $44^{\circ}$ 
The 'molecular plane' for the MBANP crystal is defined as the plane containing the directions of the charge transfer axes of the two nonequivalent molecules in the unit cell (see for example, Zyss and Chemla 1987). This plane also contains the $b$-axis as a result of the two-fold rotational symmetry and is therefore orthogonal to the ac plane. In the following, the axis along the intersection of the molecular plane with the ac plane is denoted by $z_{\mathrm{m}}$ and the axis orthogonal to $y$ and $z_{\mathrm{m}}$ by $x_{\mathrm{m}}$. The triad $\left(x_{\mathrm{m}}, y, z_{\mathrm{m}}\right)$ is a set of orthogonal axes referred to the molecular plane, while $(x, y, z)$ refer to the dielectric axes at a particular frequency.

In the case of MBANP the largest refractive index is $n_{y}$. Since the angle between the molecular charge transfer axes and the $y$ (monoclinic b) axis is $37^{\circ}$, which is consistent with the qualitative conclusion that the most easily polarized direction is along the charge transfer axis. The other two principal values, pertaining to the principal axes in the ac plane, are rather close to each other. The molecular CT axes make angles of $53^{\circ}$ with the $z_{\mathrm{m}}$ axis, so that the value of the effective refractive index in this direction is comparatively low. In the $x_{\mathrm{m}}$ direction, on the other hand, the presence of the projection of the benzene ring, at right angles to the molecular plane, tends to increase the effective refractive index. As the frequency is increased, the rotation of the dielectric axes moves the $z$-dielectric axis, corresponding to the larger of the ac plane refractive indices, into closer

Table 3. Refractive indices for YAG frequency doubling.

\begin{tabular}{lll}
\hline & $\lambda=1064 \mathrm{~nm}$ & $\lambda=532 \mathrm{~nm}$ \\
\hline MBANP & & \\
$n_{x}$ & 1.620 & 1.692 \\
$n_{y}$ & 1.725 & 1.864 \\
$n_{z}$ & 1.690 & 1.764 \\
3 -methyl MBANP & & \\
$n_{x}$ & 1.5893 & 1.6896 \\
$n_{y}$ & 1.7425 & 1.9014 \\
$n_{z}$ & 1.6505 & 1.7254 \\
$n$-methyl MBANP & & \\
$n_{x}$ & & \\
$n_{y}$ & 1.5361 & 1.5642 \\
$n_{z}$ & 1.5786 & 1.6389 \\
\pm MBANP & 1.9303 & 2.1772 \\
$n_{x}$ & & \\
$n_{y}$ & & 1.792 \\
$n_{z}$ & 1.692 & 1.760 \\
\hline
\end{tabular}

All refractive indices are referred to the principal dielectric axes at the wavelength concerned. alignment with the $z_{\mathrm{m}}$ axis (see figure $3 \mathrm{a}$ ). This would be expected since the higher frequency is closer to resonance with the dominant $\mathrm{CT}$ axis transition, the ac projection of which should be polarized along $z_{\mathrm{m}}$.

In the case of $n$-methyl MBANP the angles between the CT axes of the two molecular components of the asymmetric unit and the $y$ axis are $80^{\circ}$ and $65^{\circ}$. The largest contribution from CT axis polarization is, therefore, in the ac plane. There are now two 'molecular planes', the projections of which onto ac make an angle of approximately $15^{\circ}$ with each other. The direction of the $z$ dielectric axis (which corresponds to the largest refractive index) approximately bisects this angle. The other two refractive indices, $n_{x}$ and $n_{y}$ are much smaller at all optical frequencies. There is no observable rotation of the axes.

The presence of large angular dispersion in MBANP but not in $n$-methyl MBANP is related to the much smaller difference between the $n_{x}$ and $n_{z}$ values in the former as compared to the latter. To verify this, consider a case where, at some frequency, there is an accidental coincidence of the two ac plane refractive indices in a monoclinic crystal. At this frequency the crystal would behave as though it were uniaxial and the dielectric tensor would be diagonal with respect to any choice of axes in the ac plane. A small change in frequency would now induce a small difference between $n_{x}$ and $n_{z}$ and the directions of the axes would become fixed in some position. Clearly, near the quasi-uniaxial condition the direction of the dielectric axes will be very sensitive to small changes, since at the position of equality their direction is not defined. The phenomenon of the rotation of the axes must therefore be associated with low birefringence for light polarized in the ac plane. MBANP seems to be the only nonlinear optic organic crystal hitherto investigated that satisfies this condition. In

Table 4. MBANP d-matrix (referred to $\left(x_{\mathrm{m}}, y, z_{\mathrm{m}}\right)$ coordinate system (see text)).

$\begin{array}{crrrrr}\text { CNDOVSB } & & & & & \\ 0.0000 & 0.0000 & 0.0000 & 2.6954 & 0.0000 & -7.8932 \\ -12.8290 & 62.3263 & 27.7630 & 0.0000 & 3.4975 & 0.0000 \\ 0.0000 & 0.0000 & 0.0000 & 26.5082 & 0.0000 & 2.9357\end{array}$

Two-state model $\quad \beta_{0}=14.00$

$\begin{array}{rrrrrr}0.0000 & 0.0000 & 0.0000 & 0.4011 & 0.0000 & 0.0021 \\ 0.0006 & 55.1800 & 22.6702 & 0.0000 & 0.1176 & 0.0000 \\ 0.0000 & 0.0000 & 0.0000 & 21.0528 & 0.0000 & 0.1092\end{array}$

Experimental

$\begin{array}{lrllll}0 & 0 & 0 & ? & 0 & ? \\ ? & 45 & ? & 0 & ? & 0 \\ 0 & 0 & 0 & ? & 0 & ?\end{array}$

The tensors are referred to the 'molecular plane' coordinate system. The value of $\beta_{0}$ is given in $\mathrm{cm}^{5}$ esu. The $d$-matrix coefficients are in $\mathrm{pmV}^{-1}$. 
$n$-methyl MBANP, on the other hand, there is a very large difference between $n_{x}$ and $n_{z}$ at all frequencies.

In the two orthorhombic crystals the relative magnitudes of the refractive index components are also explicable in terms of the angles made by the $\mathrm{CT}$ axes, as the most polarizable molecular directions, with the orthorhombic crystal (dielectric) axes.

\subsection{Second-harmonic generation: Theory}

The experimental section has given a summary of the experimental results for frequency doubling for the MBANP family of crystals. In this section an attempt is made to rationalize the results using what has come to be regarded as a standard approach for the theoretical assessment of nonlinear optic organic crystals. The procedure is to take a quantum theoretic calculation of the hyperpolarizability tensor of the molecules and then to employ the oriented gas model to combine the contributions of the molecules and calculate the crystal susceptibility tensors (see, for example, Zyss and Chemla 1987). The oriented gas model uses internal field factors in forms which have been deduced by considering cavities in continuous and fairly dilute media, ideally gases. Both parts of this procedure are open to criticism. The quantum calculations, while they may be adequate to give a comparison of the overall strength of the nonlinearity in different molecules, are of questionable accuracy when it comes to predicting the details of the hyperpolarizability tensor. Furthermore the molecule in the crystal is perturbed by strong permanent crystal fields which possibly somewhat modify its electronic structure, an effect which is distinct from the induced internal field effect. The cavity model is evidently a rather crude approximation for the internal fields. Nevertheless, the approach is widely used and its results often quoted with confidence, with a tacit assumption that it will always get the right

Table 5. 3-methyl MBANP d-matrix (referred to orthorhombic crystal axes/dielectric axes).

\begin{tabular}{|c|c|c|c|c|c|}
\hline \multicolumn{6}{|c|}{ CNDOVSB } \\
\hline 0.0000 & 0.0000 & 0.0000 & $15 \cdot 2197$ & 0.0000 & 0.0000 \\
\hline 0.0000 & 0.0000 & 0.0000 & 0.0000 & 18.6305 & 0.0000 \\
\hline 0.0000 & 0.0000 & 0.0000 & 0.0000 & 0.0000 & $15 \cdot 3275$ \\
\hline \multicolumn{6}{|c|}{ Two-state model $\beta_{0}=15.4$} \\
\hline 0.0000 & 0.0000 & 0.0000 & 8.7429 & 0.0000 & 0.0000 \\
\hline 0.0000 & 0.0000 & 0.0000 & 0.0000 & 9.0877 & 0.0000 \\
\hline 0.0000 & 0.0000 & 0.0000 & 0.0000 & 0.0000 & 8.5868 \\
\hline \multicolumn{6}{|c|}{ Experimental } \\
\hline 0 & 0 & 0 & 19 & 0 & 0 \\
\hline 0 & 0 & 0 & 0 & 22 & 0 \\
\hline 0 & 0 & 0 & 0 & 0 & 23 \\
\hline
\end{tabular}

The value of $\beta_{0}$ is given in $\mathrm{cm}^{5}$ esu. The d-matrix coefficients are in $\mathrm{pmV}^{-1}$. results. It, therefore, seems desirable to try to test the method on the well characterized MBANP family.

Even within the limitations of the simplified theory there are a number of variants. The simplest version, referred to here as the two-state model assumes that the entire nonlinear response of the molecule is related to movement of charge along one axis-the charge transfer axis (CT axis). This idea is equivalent to assuming that the hyperpolarizability arises from one virtual electronic transition, which is polarized along the CT axis. In donor/acceptor molecules, like MBANP and its derivatives, this axis is approximately along the donor-acceptor axis, which is usually also the direction of the molecular dipole in both the ground and the important excited state. Here we have taken the $\mathrm{CT}$ axis, in all the molecules, along the line joining the $-\mathrm{NO}_{2}$ and amino nitrogen atoms. This choice is a little arbitrary, but is in the spirit of the simple model commonly used. The molecular hyperpolarizability in a molecular coordinate system in which, say, the CT axis is the $X$-axis, has only one nonzero element, which can be represented as:

$$
\beta_{X X X}=\beta_{0} .
$$

The transformation to crystal coordinates involves only the direction cosines $l_{i X}$ of the CT axis with an orthogonal set of axes $(i=x, y, z)$ fixed in the crystal. It is then possible to obtain a hyperpolarizability, $B_{i j k}$, for the unit cell by using the equation,

$$
B_{i j k}=\sum_{n} l_{i X}^{(n)} l_{j X}^{(n)} l_{k X}^{(n)} \beta_{0},
$$

where the sum is over the molecules in one unit cell (Zyss and Oudar 1982; Zyss and Chemla 1987).

There are a number of quantum chemistry programmes, that contain procedures for computing the full hyper-

Table 6. $n$-methyl MBANP d-matrix (referred to dielectric axes $(x, y, z)$. Axes rotated about b by $44^{\circ}$ from piezoelectric

\begin{tabular}{|c|c|c|c|c|c|}
\hline \multicolumn{6}{|c|}{ CNDOVSB } \\
\hline $0 \cdot 0000$ & 0.0000 & $0 \cdot 0000$ & 3.0031 & 0.0000 & 0.1805 \\
\hline-0.2416 & -0.5672 & $40 \cdot 8835$ & 0.0000 & 2.8527 & 0.0000 \\
\hline 0.0000 & 0.0000 & 0.0000 & 49.0640 & 0.0000 & 3.4322 \\
\hline \multicolumn{6}{|c|}{ Two-state model $\beta_{0}=19.00$} \\
\hline 0.0000 & 0.0000 & 0.0000 & 2.6832 & 0.0000 & 0.4357 \\
\hline 0.4456 & 3.0900 & 32.9984 & 0.0000 & 2.7442 & 0.0000 \\
\hline 0.0000 & 0.0000 & 0.0000 & 37.2345 & 0.0000 & 3.0965 \\
\hline \multicolumn{6}{|c|}{ Experimental } \\
\hline 0 & 0 & 0 & 5.9 & 0 & $2 \cdot 4$ \\
\hline$\sim 0$ & 4.4 & 49 & 0 & -15 & 0 \\
\hline 0 & 0 & 0 & -9.7 & 0 & $\sim 0$ \\
\hline
\end{tabular}
system).

The value of $\beta_{0}$ is given in $\mathrm{cm}^{5}$ esu. The $\mathbf{d}$-matrix coefficients are in $\mathrm{pmV}^{-1}$. 
polarizability tensor. $A b$ initio packages are being used increasingly, but most existing work on organics has used semi-empirical quantum theory in one or other of two general types of procedure. In the first, the finite field method, the molecule is perturbed by a system of fixed charges set up around it and a new ground state energy and dipole calculated. Coupled Hartree-Fock perturbation theory is often employed, as an alternative to a purely numerical approach, to determine the perturbed ground state. The hyperpolarizability is calculated from derivatives with respect to the fields. In the second approach, the SOS method, time-dependent perturbation theory involving a sum over virtual excited states is employed. The calculation is parametrized to reproduce reasonable excited state energies and dipoles as determined by standard UV/visible spectroscopy. Here we adopt a version of the SOS approach (CNDOVSB (Docherty et al 1985; Pugh and Morley 1987)) which has been widely used. A survey and comparison of the results obtained from other packages applied to the MBANP family would be of interest but has not yet been attempted. In the CNDOVSB method a set of 50 singly excited configurations has been used in each case and the full $\beta_{i j k}$ tensor calculated. The calculation has been carried out on molecules taken from the crystallographic atomic coordinate files without change of orientation. It is then a simple matter, using the symmetry operators which relate the nonequivalent molecules in the unit cell to find the unit cell polarizability, $\mathbf{B}$.

The oriented gas procedure (Zyss and Chemla 1987) is applied in the same way to the B tensor obtained from either the two state model or the CNDOVSB model. The Lorentz-Lorenz field factors transform as second-rank tensors with the same principal axes as the dielectric tensor. Their principal values, which are referred to the dielectric axes are calculated from the principal refractive indices as,

Table 7. \pm MBANP d-matrix (referred to orthorhombic crystal/ dielectric axes).

\begin{tabular}{|c|c|c|c|c|c|}
\hline \multicolumn{6}{|c|}{ CNDOVSB } \\
\hline 0.0000 & 0.0000 & 0.0000 & 0.0000 & 12.0736 & 0.0000 \\
\hline 0.0000 & 0.0000 & 0.0000 & 3.6759 & 0.0000 & 0.0000 \\
\hline $10 \cdot 8868$ & $4 \cdot 2891$ & -2.7587 & 0.0000 & 0.0000 & 0.0000 \\
\hline \multicolumn{6}{|c|}{ Two-state model $\quad \beta_{0}=12.3$} \\
\hline 0.0000 & 0.0000 & 0.0000 & 0.0000 & 7.3275 & 0.0000 \\
\hline 0.0000 & 0.0000 & 0.0000 & $6 \cdot 3500$ & 0.0000 & 0.0000 \\
\hline 7.0809 & $6 \cdot 1779$ & 0.5434 & 0.0000 & 0.0000 & 0.0000 \\
\hline \multicolumn{6}{|c|}{ Experimental } \\
\hline 0.0000 & 0.0000 & 0.0000 & 0.0000 & ? & 0.0000 \\
\hline 0.0000 & 0.0000 & 0.0000 & $?$ & 0.0000 & 0.0000 \\
\hline 6.8 & 4.7 & 0.84 & 0.0000 & 0.0000 & 0.0000 \\
\hline
\end{tabular}

The value of $\beta_{0}$ is given in $\mathrm{cm}^{5}$ esu. The d-matrix coefficients are in $\mathrm{pmV}^{-1}$.

$$
f_{i}^{\omega}=\frac{\left(n_{i}^{\omega}\right)^{2}+2}{3}
$$

In every case except MBANP the rest of the calculation can be carried out in the same fixed principal dielectric axis coordinate system, so that no transformation of the Lorentz factor tensor is necessary. In the case of MBANP because of the rotation of the axes the calculation has to be performed more generally. For any set of orthogonal axes the second-order susceptibility is given in the oriented gas model by,

$$
\chi_{i j k}(-2 \omega ; \omega, \omega)=\frac{f_{i i^{\prime \prime}}^{2 \omega} f_{j j^{\prime}}^{\omega} f_{k k^{\prime}}^{\omega}}{\varepsilon_{0} V_{\text {cell }}} B_{i j^{\prime} k^{\prime}},
$$

where summation over the repeated indices $\left(i^{\prime}, j^{\prime}, k^{\prime}=x, y, z\right)$ is implied. The field factor tensor at each frequency is found by performing the appropriate rotation operations between the dielectric frame for that frequency and the common frame of (5). If the hyperpolarizabilities are expressed (as they often are) in $\mathrm{cm}^{\mathrm{s}}$ esu and the susceptibilities in $\mathrm{pmV}^{-1}$, then, with the cell volume in (Angstrom) ${ }^{3}$ the equation can be written in a useful form for computation as:

$$
\chi_{i j k} / \mathrm{pmV}^{-1}=419 \cdot 0 \frac{f_{i i^{\prime}}^{2 \omega} f_{j^{\prime}}^{\omega} f_{k k^{\prime}}^{\omega}}{\left(V_{\text {cell }} / \text { Angstrom }{ }^{3}\right)}\left(B_{l^{\prime} k^{\prime}} / \mathrm{cm}^{5} \text { esu }\right)
$$

For phase-matched SHG, a direction of propagation that lies on the phase matching locus must be chosen. For each such direction there is an effective phase matching coefficient given by,

$$
d_{\text {eff }}=e_{i}^{2 \omega} e_{j}^{\omega} e_{k}^{\omega} \chi_{i j k}^{(2)}
$$

where the $\mathbf{e}$ 's are the unit polarization vectors of the fundamental and second-harmonic phase-matched fields propagating in the chosen direction. If complete refractive index data is available at both frequencies, the phase matching locus and the e vectors can be calculated, so that if the components of the $\chi_{i j k}^{(2)}$ tensor have also been found, either from Maker fringe measurements or by calculation, $d_{\text {eff }}$ can be determined. An experimental value of $d_{\text {eff }}$ can be obtained from the peak phase matching intensity at any point on the locus.

\subsection{Second-harmonic generation: Analysis of results}

3.3a MBANP: The second-order tensor is referred to the 'molecular plane' system of axes $\left(x_{\mathrm{m}}, y, z_{\mathrm{m}}\right)$. It then follows that in the two-state model all components of $\chi^{2}$ in which $x_{\mathrm{m}}$ is a coordinate index are zero. The computed tensors, referred to the molecular axes are given in table 4 . 
Table 4 shows that the two-state and CNDOVSB calculations identify the three largest elements in the same order. In the CNDOVSB calculation the elements related to an $x_{\mathrm{m}}$-polarized field are substantially smaller than the three major elements but are still appreciable. In particular the value of the $d_{21}$ element is quite at variance with the predictions of the two-state model. This element contains a large contribution in the sum over states calculation from $\sigma-\pi$ transitions, whereas the only nonzero molecular hyperpolarizabilty component in the two-state model is in the plane of the pyridine ring and can account only for the effect of $\pi-\pi$ transitions. The experimental value of $d_{22}=45 \mathrm{pmV}^{-1}$ is about $25 \%$ lower than the CNDO value. The value of $\beta_{0}$ used in the two-state model has been taken as the vector part of the CNDOVSB tensor. This procedure has the advantage that it can be applied uniformly in all the
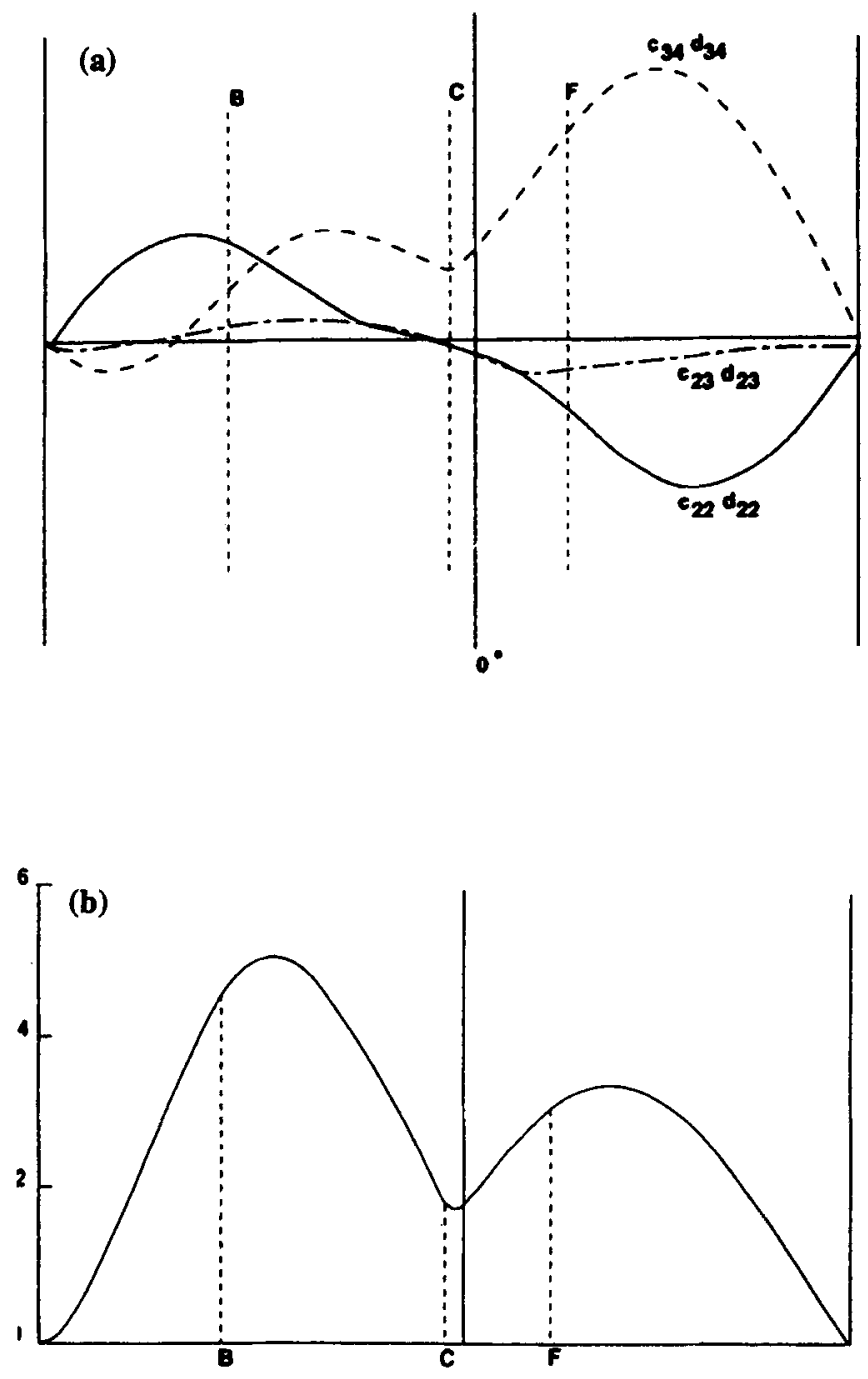

Figure 6. (a) Contributions of the individual d-matrix coefficients to $d_{\text {eff }}$ along the phase matching locus. The end points correspond to the points $A$ and $G$ in figure 3 . The solid line is the contribution of the $d_{22}$ element and (b) the effective phase matching susceptibility, $d_{\text {eff }}$ (sum of contributions shown in figure 6a). calculations and leads to a slightly higher value for the $n$-methyl compound, which would be expected from the molecular structure. If the experimental electric-field induced second-harmonic (EFISH) solution measurements on the MBANP molecule were used instead, a lower value of about $12 \mathrm{~cm}^{5}$ esu would be appropriate. This would bring the two-state model into closer agreement with the experimental value. Given the uncertainties associated with solvent effects in the EFISH technique and the rather crude approximations used in the calculations, the agreement in the absolute values is not unacceptable.

For the reasons previously noted there are no experimental determinations of the other separate elements of the $d$-matrix. The effective phase matching susceptibility, however, includes contributions from a number of elements. From the calculated tensors using the experimental refractive index and dielectric axis data it is possible to calculate $d_{\text {eff }}$ as a function of position on the phase matching locus. The results are shown in figure 6 . The reference points along the locus can be identified from figure 3 . Figure 6a shows the contributions of the individual elements of the d-matrix referred to the molecular plane coordinate system. An unusual feature is that one of the major contributors is the diagonal $d_{22}$ element. It is often stated that this type of element cannot contribute to phase matching since the matched fundamental and second-harmonic fields are orthogonal. In biaxial crystals the two fields are strictly orthogonal only when both are propagating in a principal dielectric plane. Where there is no dispersion of the axes deviations from this condition tend to be small whatever the direction of propagation. The rotation of $26^{\circ}$ between the directions of the fundamental and doubled dielectric axes in MBANP invalidates this result. The predicted maximum $d_{\text {eff }}$ is $6 \mathrm{pmV}^{-1}$, close to the experimental result, and the position of the maximum on the locus is also well reproduced. Experimentally the signal is found to fall off on either side of a maximum at the point in the region between points $\mathrm{B}$ and $\mathrm{C}$ on the locus shown in figure 3.

3.3b 3-methyl MBANP: The calculated and experimental d-matrices are shown in table 5. The agreement between the CNDOVSB values and experiment is very satisfactory, but the two-state model gives matrix elements which are only about half as great as those observed. The size of these elements, determined experimentally and by CNDOVSB, relative to $d_{22}$ for MBANP are in agreement with the general qualitative ideas that are encapsulated in the two-state model, but the better quantitative agreement with the CNDOVSB calculation is indicative of contributions that cannot be accounted for within the two-state model.

3.3c N-methyl MBANP: The asymmetric unit consists of two molecules and the projection of the 'molecular planes' defined by these two entities onto the ac plane 
gives two lines at a small angle with each other. The $z$-dielectric axis lies in a direction between these two lines. The behaviour of the two-state model found in MBANP where all the components involving an $x_{\mathrm{m}}$ index vanished is, therefore, reproduced only approximately in this case. The two calculations are in reasonable agreement with each other and both predict that the $y z z$ and $z y z$ elements $\left(d_{23}\right.$ and $\left.d_{34}\right)$, equal according to Kleinman symmetry, are much larger than the others. While the magnitude of the former is well reproduced, especially by the CNDOVSB calculation, there is a major discrepancy between theory and experiment with respect to the $d_{34}$ element. The experimental result here is rather surprising since the violation of Kleinman symmetry means that the reversal of sign of one of the elements must appear only at finite frequency, which is not easily explicable unless the second-harmonic photon energy exceeds one of the allowed transition energies.

$3.3 \mathrm{~d} \pm$ MBANP: A reduction in the magnitude of the larger elements would be expected to result from the high degree of cancellation of the noncentrosymmetric effects produced by the arrangement of the eight molecules in the unit cell. Such an effect is indeed found, both in the theoretical and experimental results. The agreement between the predictions of the CNDOVSB theory and the three experimental results is reasonable and the two-state theory, in this case, is in very good agreement with the experiments. The latter result has already been reported by Kondo et al (1995).

\section{Conclusions}

In each crystal the relative magnitude of the three principal refractive indices and directions of the dielectric axes can be qualitatively related to the molecular structure. While calculations of refractive indices of sufficient accuracy to be of use in the prediction, for example, of phase matching loci do not seem to be possible, in all of the cases considered here there is a correlation between the identity of the largest refractive index component and the direction of the largest projection of the molecular charge transfer axes. The existence of rotation of the axes in MBANP, but not in the other monoclinic crystal, $n$-methyl MBANP, can be associated with the comparatively small difference between the ac plane refractive indices in the former.

With certain reservations, the second-harmonic generation d-matrices can be claimed to be in, at least, semiquantitative agreement with experiment, both in relation to the comparative size of the major elements in the different crystals and with respect to the relative sizes of the elements within each matrix. A major discrepancy between theory and experiment occurs in the case of $d_{34}$ element in $n$-methyl MBANP. Also, some of the smaller elements are not well reproduced, but since these often arise from small differences between the much larger contributions of the individual molecules in the unit cell, it is not surprising that the agreement should be poorer.

Applications of the kind of theoretical approach described here should not, realistically, be expected to give more than a rough guide to the significant features of the crystal d-matrix. However, the general success of the approach at this level would indicate that some particular explanation should be sought for the occasional gross discrepancy, as in the case of the $n$-methyl MBANP $d_{34}$ element. With this exception the theory, within its limitations, gives an adequate account of the MBANP family of crystals.

\section{References}

Bailey R T et al 1988a Opt. Commun. 65229

Bailey R T et al 1988b J. Mod. Opt. 35511

Bailey R T et al 1988c Proc. SPIE 971 p. 76

Bailey R T, Cruickshank F R, Pugh D and Sherwood J S 1991 a Acta Crystallogr. A47 145

Bailey R T, Cruickshank F R, Pavlides P, Pugh D and Sherwood J S 1991b J. Phys. D: Appl. Phys. 24135

Bailey R T, Cruickshank F R, Pugh D and Sherwood J S 1993 J. Phys. D: Appl. Phys. 26 B208

Cheng Y Y, Crowley J I, Jain K and Tweig R J 1982 IBM Tech. Disclosure Bull. 251625

Docherty V J, Morley J O and Pugh D 1985 J. Chem. Soc. Faraday Trans. 811179

Kondo T, Ogasawara N, Umegakai S and Ito R 1988a Proc. SPIE 971 p. 83

Kondo $\mathrm{T}$, Ogasawara $\mathrm{N}$, Ito $\mathrm{R}$, Ishida $\mathrm{K}$, Tanase $\mathrm{T}$, Murata $\mathrm{T}$ and Hidai M 1988b Acta Crystallogr. C44 102

Kondo T, Morita R, Ogasawara N, Umegakai S and Ito R 1989 Jpn J. Appl. Phys. 281622

Kondo T, Akase F, Kumagai M and Ito R 1995 Opt. Rev. 2 128

Lochran S, Bailey R T, Cruickshank F R, Pugh D, Sherwood J N, Simpson G S, Wallis J D and Langley P J 1998a J. Phys. Chem A. (in press)

Lochran S, Bailey R T, Cruickshank F R, Pugh D, Sherwood J N, Wallis J D and Langley P J 1998b Proc. SPIE (in press)

Pugh D and Morley J O 1987 in Nonlinear optical properties of organic molecules and crystals (eds) D S Chemla and J Zyss Ch. II Vol. 1 p. 193

Tweig R J and Dirk C W 1986 IBM Report RJ 5237154077

Tweig R J, Azema A, Jain K and Cheng Y Y 1982a Chem. Phys. Lett., 92208

Tweig R J, Jain K and Cheng Y Y, Crowley J I and Azema A 1982b Polymer Reprints 23147

Zyss J and Oudar J L 1982 Phys. Rev. A26 2028

Zyss J and Chemla D S 1987 in Nonlinear optical properties of organic molecules and crystals (eds) D S Chemla and J Zyss Ch. II, Vol. 1, p. 23 